\title{
DOES DRONEDARONE IMPROVE THIOL-SPECIFIC OXIDATIVE STRESS? EXPERIMENTAL STUDY ON HYPERTENSIVE RATS
}

L. Pazó-Sayós', R. Martín-Oropesa', P. Rodríguez-Rodríguez ${ }^{3}$, E. Delgado-Baeza², MC. González ${ }^{3}$, B. Quintana-Villamandos 1,2,4 . 'Dept. Anesthesiology and Intensive Care, Gregorio Marañón Hospital, Madrid, ${ }^{2}$ Instituto de Investigación Sanitaria Gregorio Marañón, liSGM, Madrid, ${ }^{3}$ Faculty of Medicine, Universidad Autónoma de Madrid, ${ }^{4}$ Faculty of Medicine, Universidad Complutense de Madrid.

Background and Goal Study: plasma protein thiolation index (PTI) has been studied as a biomarker of thiol-specific oxidative stress in haemodyalized patients ${ }^{1}$. On the other hand, dronedarone is an antiarrhythmic agent who acts on multiple ion channels. We hypothesized that PTI could also be an oxidative stress biomarker for left ventricular hypertrophy (LVH) and that dronedarone could improve thiol-specific oxidative stress in an experimental model of arterial hypertension and LVH.

\begin{abstract}
Materials and Methods: adult male spontaneously hypertensive (SHR) rats were randomly divided into therapy group (SHR-D, $n=9)$ and placebo group (SHR, $n=9$ ). Wistar Kyoto rats were used as normotensive controls (WKY, $\mathrm{n}=9$ ). After 14 days of treatment, blood samples were obtained by intracardiac puncture. After centrifuging them, we used the plasma to analyse the concentration of thiols and thiolated proteins. PTI was calculated as follows ${ }^{1}$ : [thiolated proteins] / [thiols]. The parameters were compared using singlefactor analysis of variance and a post hoc Bonferroni correction was applied. All data were expressed as mean \pm SEM. $\mathrm{P}<0.05$ was considered significant. All procedures were approved by the Ethics Committee of Hospital General Universitario Gregorio Marañon, Madrid, Spain.
\end{abstract}

Results and discussion: hypertensive controls showed an increase in PTI compared to normotensive controls. Interestingly, after treatment with dronedarone, there was a decrease in PTI in the SHR-D group compared to control group SHR $(\mathrm{p}<0.01)$, with no differences between SHR-D and WKY.
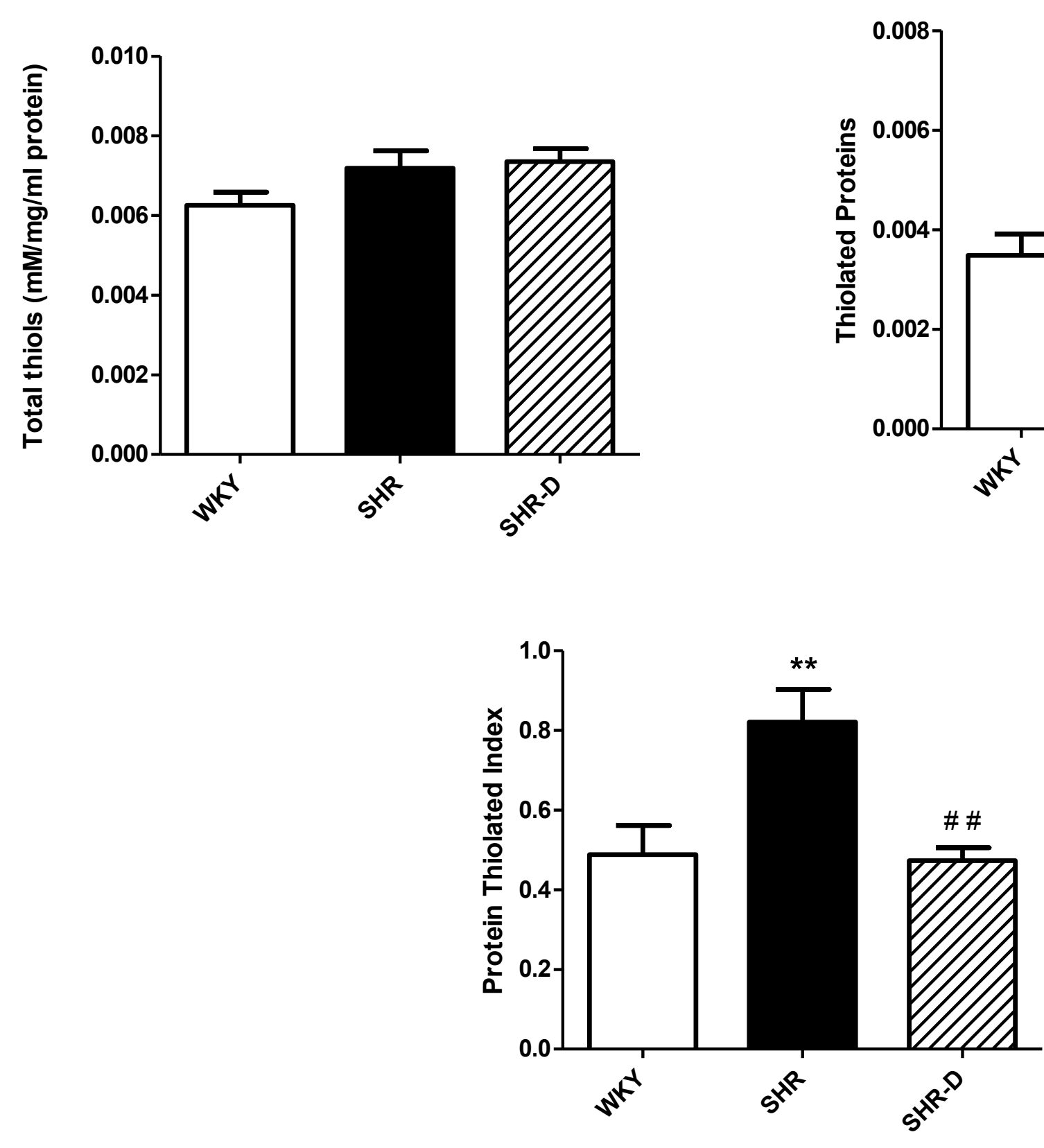

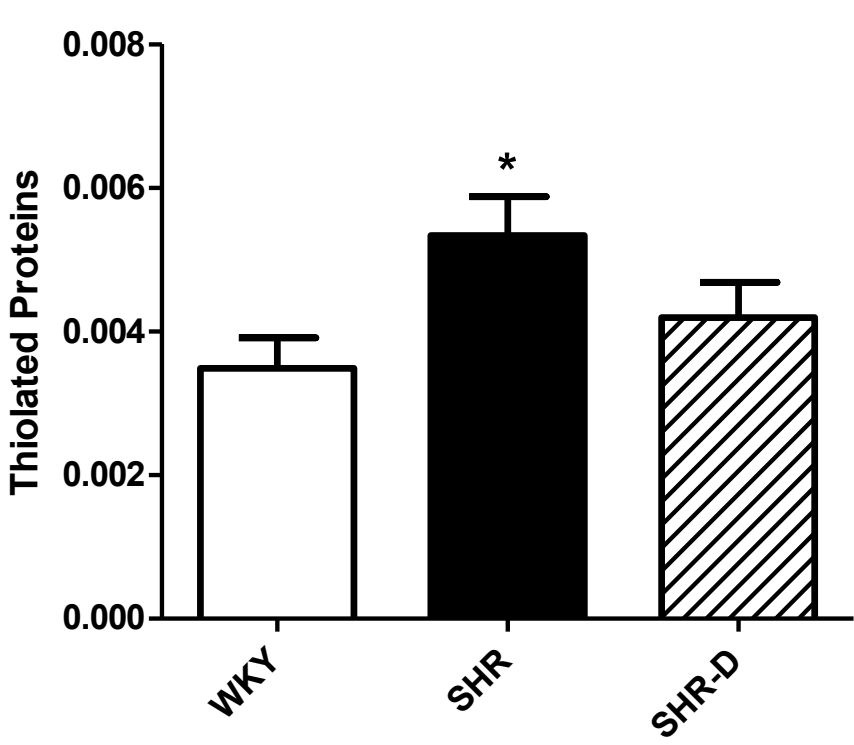

Oxidative stress biomarkers analysis in all three groups (WKY, SHR, SHR-D). A) Total thiols B) Thiolated proteins C) Protein thiolation index. Data expressed as MEAN \pm SEM. $* \mathrm{p}<0,05$ ** $\mathrm{p}>0,01$ vs. WKY. \#\# $\mathrm{p}<0,01$ vs SHR.

Conclusions: treatment with dronedarone improves thiol-specific oxidative stress in an experimental model of arterial hypertension and LVH.

\section{References:}

1. Colombo G, Reggiani F, Podestà MA, Garavaglia ML, Portinaro NM, Milzani A, et al. Plasma protein thiolation index (PTI) as a biomarker of thiol-specific oxidative stress in haemodialyzed patients.. Free Radical. Biol. Med. 2015; 89:443-451. 